\title{
What Makes the Bioactive Lipids Phosphatidic Acid and Lysophosphatidic Acid So Special?
}

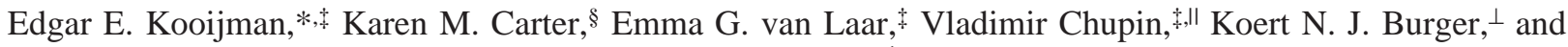 \\ Ben de Kruijff \\ Department of Biochemistry of Membranes, Bijvoet Center, Institute of Biomembranes, Utrecht University, \\ Utrecht, The Netherlands, Department of Chemistry, University of Virginia, Charlottesville, Virginia 22904, and Department of \\ Biochemical Physiology, Institute of Biomembranes, Utrecht University, Utrecht, The Netherlands
}

Received September 15, 2005

\begin{abstract}
Phosphatidic acid and lysophosphatidic acid are minor but important anionic bioactive lipids involved in a number of key cellular processes, yet these molecules have a simple phosphate headgroup. To find out what is so special about these lipids, we determined the ionization behavior of phosphatidic acid (PA) and lysophosphatidic acid (LPA) in extended (flat) mixed lipid bilayers using magic angle spinning ${ }^{31} \mathrm{P}$ NMR. Our data show two surprising results. First, despite identical phosphomonoester headgroups, LPA carries more negative charge than PA when present in a phosphatidylcholine bilayer. Dehydroxy-LPA [1-oleoyl-3-(phosphoryl)propanediol] behaves in a manner identical to that of PA, indicating that the difference in negative charge between LPA and PA is caused by the hydroxyl on the glycerol backbone of LPA and its interaction with the phosphomonoester headgroup. Second, deprotonation of phosphatidic acid and lysophosphatidic acid was found to be strongly stimulated by the inclusion of phosphatidylethanolamine in the bilayer, indicating that lipid headgroup charge depends on local lipid composition and will vary between the different subcellular locations of (L)PA. Our findings can be understood in terms of a hydrogen bond formed within the phosphomonoester headgroup of (L)PA and its destabilization by competing intra- or intermolecular hydrogen bonds. We propose that this hydrogen bonding property of (L)PA is involved in the various cellular functions of these lipids.
\end{abstract}

Phosphatidic acid (PA) ${ }^{1}$ and the related lipid lysophosphatidic acid (LPA) are important minor lipid species in the cell. They are involved in many intracellular processes, and are important intermediates in lipid biosynthesis (1). For example, binding of LPA to its receptors evokes various cellular responses, and the local formation of (L)PA is part of signaling cascades, in particular in the regulation of membrane dynamics such as fusion and fission events, either indirectly through the recruitment of downstream effectors or directly by mediating (local) changes in the biophysical properties of the membrane $(2-12)$.

PA and LPA have a relatively simple chemical structure consisting of only a glycerol, one (LPA) or two (PA) acyl

\footnotetext{
†.d.K., E.E.K., and K.N.J.B. are supported by NWO/FOM/ALW, "Fysische Biologie" program. K.N.J.B. is supported by the Human Frontier Science Organization and EC Network Grant HPRN-CT-200200259. K.M.C. is supported by NIH Grant RO1-6M527222.

* To whom correspondence should be addressed: Department of Biochemistry of Membranes, Bijvoet Center and Institute of Biomembranes, Utrecht University, Padualaan 8, $3584 \mathrm{CH}$ Utrecht, The Netherlands. Telephone: ++3130253 5511. Fax: ++31302533969. E-mail: e.e.kooijman@chem.uu.nl.

$\doteqdot$ Department of Biochemistry of Membranes, Utrecht University.

$\S$ University of Virginia.

"Current address: Shemyakin-Ovchinnikov Institute of Bioorganic Chemistry, Russian Academy of Sciences, Moscow, Russia.

${ }^{\perp}$ Department of Biochemical Physiology, Utrecht University.

${ }^{1}$ Abbreviations: dehydroxy-LPA, 1-oleoyl-3-(phosphoryl)propanediol; LPA, lysophosphatidic acid; LPC, lysophosphatidylcholine; MAS, magic angle spinning; NMR, nuclear magnetic resonance; PA, phosphatidic acid; PC, phosphatidylcholine; PE, phosphatidylethanolamine; PS, phosphatidylserine.
}

chains, and a phosphate, and it is interesting to note that these simple phospholipids are involved in such diverse processes, and are able to bind specifically to so many different types of proteins $(3,13)$. The question then is what is so special about these lipids. An obvious suggestion relates to the phosphate headgroup, which is attached to the glycerol backbone as a phosphomonoester, a unique feature of these lipids. Phosphomonoesters have two $\mathrm{p} K_{\mathrm{a}}$ 's, one of which is expected to be in the physiological $\mathrm{pH}$ range. As a consequence, small changes in (physiological) $\mathrm{pH}$ will affect the charge and influence the molecular shape and lipid phase behavior of these lipids $(14,15)$. Under physiological conditions at neutral $\mathrm{pH}$, phosphatidic acid is a cone (type II)shaped lipid with a negative spontaneous curvature close to that of unsaturated phosphatidylethanolamine (PE), whereas LPA is an inverted cone (type I)-shaped lipid with the most positive spontaneous curvature of any membrane lipid measured to date $(11,12)$. Lipid headgroup charge not only is a key determinant of the molecular shape of (L)PA but also is expected to greatly influence interactions with neighboring lipids and (L)PA-binding proteins. Clearly, insight into the charge of (L)PA and the way this charge is influenced by physiological factors such as $\mathrm{pH}$ and membrane lipid composition is essential to our understanding of the biological roles of PA and LPA.

We therefore set out to determine the charge on (L)PA in model membrane systems that are relevant to biological membranes. Lipid headgroup charge cannot easily be determined by $\mathrm{pH}$-dependent phase transition studies, surface 
potential measurements, or traditional acid-base titration methods since these methods are indirect or hampered by difficulties and uncertainties in data analysis $(16,17)$. However, because the hydroxyls on (L)PA are close to the phosphorus nucleus (protons separated from the phosphor by only an oxygen), their ionization will influence the magnetic properties of this nucleus, which can be measured by ${ }^{31} \mathrm{P}$ NMR as a pH-dependent chemical shift in highresolution ${ }^{31} \mathrm{P}$ NMR spectra. However, ${ }^{31} \mathrm{P}$ NMR spectra of extended bilayers are characterized by broad lines due to the large chemical shift anisotropy, which masks $\mathrm{pH}$ dependent changes in chemical shift. To circumvent this problem, earlier studies made use of small unilamellar vesicles (SUVs) and micelles in which the phospholipids undergo rapid isotropic motion, thereby giving rise to highresolution ${ }^{31} \mathrm{P}$ NMR spectra $(18-20)$. A disadvantage of these systems is their high curvature, which is not typical for biological membranes. With the advance of magic angle spinning (MAS) NMR, it is now possible to directly measure the chemical shift of phospholipids in biologically more relevant extended bilayer systems (21). We used this technique to determine the ionization of PA and LPA in extended bilayers composed of the abundant membrane phospholipids PC (phosphatidylcholine), PE, and their mixtures.

We show that titration curves can be measured using MAS ${ }^{31} \mathrm{P} \mathrm{NMR}$ and that $\mathrm{p} K_{\mathrm{a} 1}$ and $\mathrm{p} K_{\mathrm{a} 2}$ (and thus charge) can be determined with high accuracy. We find that, despite identical phosphate headgroups, LPA has a significantly lower $\mathrm{p} K_{\mathrm{a} 2}$ value than $\mathrm{PA}$ in pure $\mathrm{PC}$, indicating that LPA carries more negative charge than PA at physiological $\mathrm{pH}$. In mixed lipid bilayers of PC and PE, the $\mathrm{p} K_{\mathrm{a} 2}$ of LPA and PA decreases with an increase in PE content, and therefore depends critically on the composition of the surrounding zwitterionic lipids, i.e., the PC:PE ratio. A unifying mechanism will be proposed to explain these findings based on unique hydrogen bonding possibilities of the phosphomonoester headgroup.

\section{MATERIALS AND METHODS}

Sample Preparation. 1,2-Dioleoyl-sn-glycero-3-phosphoethanolamine (DOPE), 1,2-dioleoyl-sn-glycero-3-phosphocholine (DOPC), 1,2-dioleoyl-sn-glycero-3-phosphate (monosodium salt; DOPA), 1,2-dioleoyl-sn-glycero-3-phosphoserine (sodium salt; DOPS), 1-oleoyl-sn-glycero-3-phosphate (sodium salt; LPA), and 1-oleoyl-sn-glycero-3-phosphocholine (LPC) were purchased from Avanti Polar Lipids (Birmingham, AL). 1-Oleoyl-3-(phosphoryl)propanediol (dehydroxyLPA) was synthesized as reported previously (22). Lipid purity was checked by thin-layer chromatography and judged to be $>99 \%$. Water used in the experiments came from a Milli-Q system (Millipore, Bedford, MA) and had a resistivity of $18.2 \mathrm{M} \Omega \mathrm{cm}$.

NMR samples, for $\mathrm{pH}$ titration purposes, were prepared by mixing appropriate amounts of lipid stock [concentration determined by a $\mathrm{P}_{\mathrm{i}}$ determination (23)] from chloroform and methanol, subsequently dried under a stream of $\mathrm{N}_{2}$, and placed under high vacuum overnight. Samples were hydrated using an appropriate buffer. The following buffers were used: $100 \mathrm{mM} \mathrm{KCl}-\mathrm{HCl}$ for $\mathrm{pH} 1.5-2.5,10 \mathrm{mM}$ Hepes, $20 \mathrm{mM}$ Mes, $30 \mathrm{mM}$ citric acid- $\mathrm{NaOH}$, and $100 \mathrm{mM} \mathrm{NaCl}$ for $\mathrm{pH}$ 2.5-7.5, $50 \mathrm{mM}$ Tris- $\mathrm{HCl}$ and $100 \mathrm{mM} \mathrm{NaCl}$ for
pH $7.5-9.0$, or $50 \mathrm{mM}$ glycine- $\mathrm{NaOH}$ and $100 \mathrm{mM} \mathrm{NaCl}$ for $\mathrm{pH}$ 9.0-10.5. Each contained $2 \mathrm{mM}$ EDTA to complex any traces of divalent cations. The samples were then subjected to a minimum of two freeze-thaw-vortex cycles, after which the $\mathrm{pH}$ of the samples was measured. This $\mathrm{pH}$, measured after lipid hydration, was used to construct the $\mathrm{pH}$ titration curves. NMR samples for measurement at constant $\mathrm{pH}$ were prepared as described above and hydrated with a buffer containing $100 \mathrm{mM}$ Hepes, $5 \mathrm{mM}$ acetic acid-NaOH, $100 \mathrm{mM} \mathrm{NaCl}$, and $2 \mathrm{mM}$ EDTA (pH 7.2). After hydration, the $\mathrm{pH}$ of these lipid dispersions was found to be generally within $0.05 \mathrm{pH}$ unit of $\mathrm{pH} 7.2$; in some cases, the $\mathrm{pH}$ was adjusted by the addition of $\mathrm{NaOH}$ or $\mathrm{HCl}$ to come within this range. The lipid dispersions were concentrated in a tabletop centrifuge $(70000 \mathrm{rpm}$ for $45 \mathrm{~min}$ at room temperature), and the (wet) lipid pellet was transferred to $4 \mathrm{~mm}$ $\mathrm{TiO}_{2}$ MAS NMR sample tubes.

NMR. ${ }^{31} \mathrm{P}$ NMR spectra were recorded on a Bruker Avance (Karlsruhe, Germany) 500 widebore spectrometer at 202.48 $\mathrm{MHz}$, using a $4 \mathrm{~mm}$ cross-polarization (CP) MAS NMR probe. Samples were spun at the magic angle $\left(54.7^{\circ}\right)$ at 5 $\mathrm{kHz}$ to average the chemical shift anisotropy, and the chemical shift position of (L)PA was recorded relative to $85 \% \mathrm{H}_{3} \mathrm{PO}_{4}$. Under stable spinning conditions, typically 100-1000 scans were recorded depending on the amount of lipid recovered in the pellet. Static spectra were recorded by the spin-echo technique with proton decoupling in a 4 mm CP MAS NMR probe to check the lipid phase of the samples, where appropriate. Experiments were carried out at $20.0 \pm 0.5^{\circ} \mathrm{C}$.

Determination of $p K_{a}$ Values. The $\mathrm{p} K_{\mathrm{a}}$ values for (L)PA were determined by using a relation derived from the Henderson-Hasselbach equation (24) and a nonlinear leastsquares fit.

$$
\delta=\frac{\delta_{\mathrm{AB}}+\delta_{\mathrm{AA}} \times 10^{\mathrm{p} K_{\mathrm{a} 1}-\mathrm{pH}}+\delta_{\mathrm{BB}} \times 10^{\mathrm{pH}-\mathrm{p} K_{\mathrm{a} 2}}}{1+10^{\mathrm{p} K_{\mathrm{a} 1}-\mathrm{pH}}+10^{\mathrm{pH}-\mathrm{p} K_{\mathrm{a} 2}}}
$$

$\delta_{\mathrm{AA}}, \delta_{\mathrm{AB}}$, and $\delta_{\mathrm{BB}}$ are the chemical shifts of the associated, singly dissociated, and doubly dissociated state, respectively. $\delta$ is the measured chemical shift. $\mathrm{pH}$ is the $\log$ of the measured hydrogen concentration, and $\mathrm{p} K_{\mathrm{a} 1}$ and $\mathrm{p} K_{\mathrm{a} 2}$ are the dissociation constants.

In cases where only the top part of the titration curve was measured ( $\mathrm{pH} 4-10)$, the data were fitted with a modified equation now containing only one dissociation constant (24):

$$
\delta=\frac{\delta_{\mathrm{A}} \times 10^{\mathrm{p} K_{\mathrm{a}}-\mathrm{pH}}+\delta_{\mathrm{B}}}{1+10^{\mathrm{p} K_{\mathrm{a}}-\mathrm{pH}}}
$$

Note that this barely influences the determination of the second $\mathrm{p} K_{\mathrm{a}}$ of (L)PA. Fitting the data for a full titration curve [e.g., (L)PA in DOPC] over this $\mathrm{pH}$ range (4-10), using eq 2 instead of eq 1 , did not change the value of $\mathrm{p} K_{\mathrm{a} 2}$ by more than $0.2 \%$ (data not shown).

\section{RESULTS}

MAS ${ }^{31} \mathrm{P}$ NMR was used to measure the dissociation constants of PA and LPA present at low concentrations in dispersions of PC and PE. The phospholipids used in this study contained unsaturated (oleoyl, 18:1) chains since these 


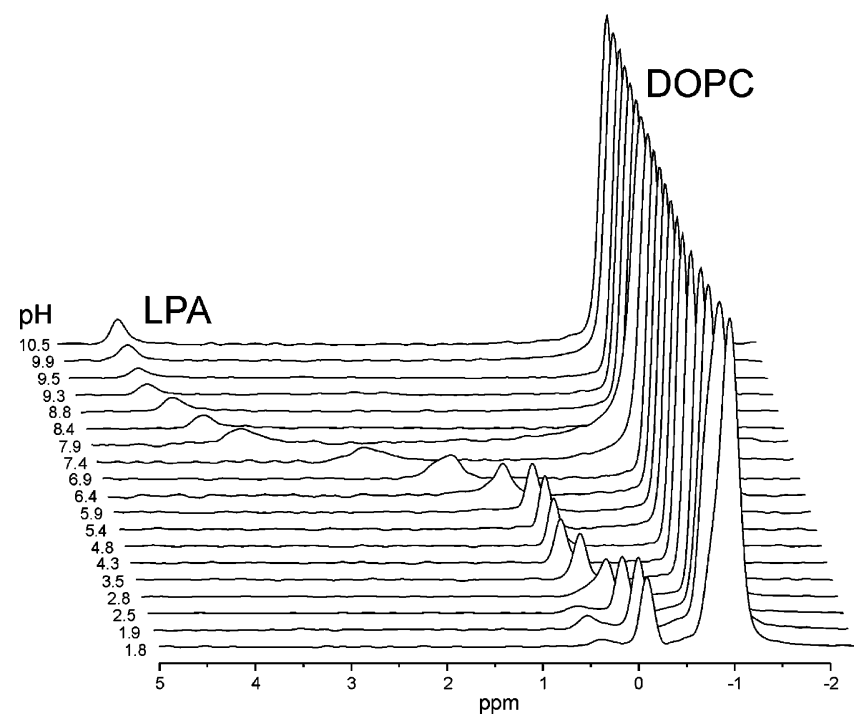

FIGURE 1: Solid-state MAS ${ }^{31} \mathrm{P}$ NMR spectra for $10 \mathrm{~mol} \%$ LPA in DOPC as a function of $\mathrm{pH}$.

give rise to fluid bilayers representative of biological membranes. All experiments were carried out in $100 \mathrm{mM}$ salt to mimic physiological conditions, and all measurements were recorded at $20^{\circ} \mathrm{C}$ to eliminate temperature effects on ionization and ${ }^{31} \mathrm{P}$ chemical shift.

Dissociation Constants of $(L) P A$ in PC Bilayers. We first determined the dissociation constants of LPA and PA in PC bilayers. Figure 1 shows representative MAS ${ }^{31} \mathrm{P}$ NMR spectra for $10 \mathrm{~mol} \%$ LPA in DOPC as a function of $\mathrm{pH}$. The individual peaks of LPA and PC are well resolved as the minor low-field peak and the large high-field peak, respectively. The $\mathrm{pH}$-dependent protonation of the phosphate headgroup resulted in a large downfield shift of the LPA peak. The titration behavior indicated the presence of two $\mathrm{p} K_{\mathrm{a}} \mathrm{s}$, as anticipated for this phosphomonoester headgroup. The nearly constant position of the PC peak demonstrates that the charge on the phosphate group of PC did not change, according to the expectation for this zwitterionic headgroup (17). A similar behavior was found for the position of the LPC and PE peak in the PE/LPC bilayer (see below), except that the PE peak moved slightly to downfield values at high $\mathrm{pH}(\mathrm{pH}>9.0)$, reflecting the onset of deprotonation of the primary amine of the PE headgroup (data not shown). At very low $\mathrm{pH}$ values, a third minor $(<1-2 \%)$ peak downfield of LPA was present due to a small amount of lipid hydrolysis at this very low $\mathrm{pH}$. Similar spectra as a function of $\mathrm{pH}$ were recorded for $10 \mathrm{~mol} \% \mathrm{PA}$ in DOPC (spectra not shown).

The chemical shifts of LPA and PA were plotted as a function of $\mathrm{pH}$ to yield the titration curves shown in Figure 2. The double-sigmoidal shape of the titration curves reflects the sequential dissociation of two protons from the phosphate headgroup. The first dissociation occurs at very low $\mathrm{pH}$ $(2<\mathrm{pH}<4)$, whereas the second dissociation occurs in a more physiological $\mathrm{pH}$ range $(6<\mathrm{pH}<9)$. The actual $\mathrm{p} K_{\mathrm{a}}$ 's were determined as described in Materials and Methods, and the results are given in Table 1 . These data show that $\mathrm{p} K_{\mathrm{a}}$ 's and in particular the more physiologically relevant $\mathrm{p} K_{\mathrm{a} 2}$ can be determined with high accuracy in extended bilayers using MAS ${ }^{31} \mathrm{P}$ NMR. Interestingly, despite identical phosphomonoester headgroups, the $\mathrm{p} K_{\mathrm{a} 2}$ of $\mathrm{PA}$ is $0.45 \mathrm{pH}$ unit higher than that of LPA in a PC bilayer. This charge difference

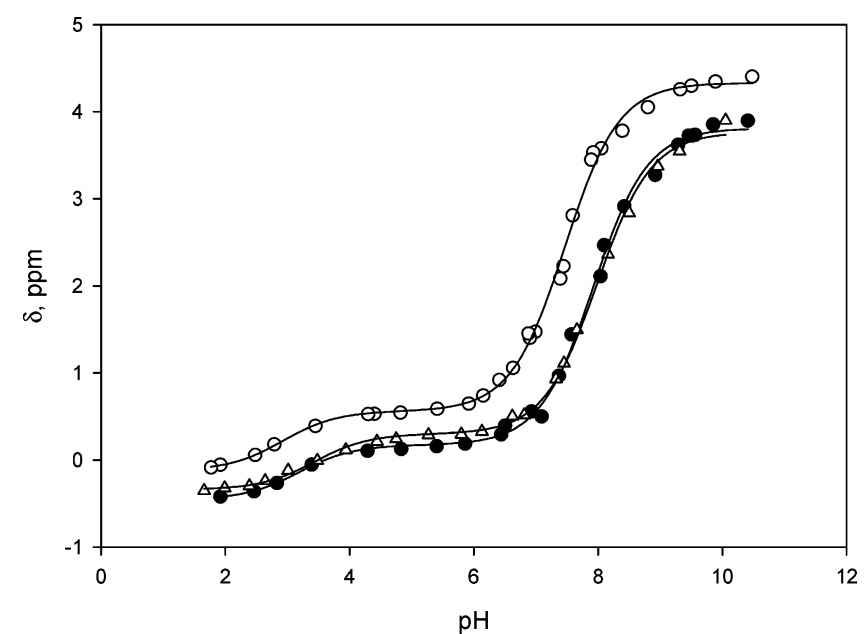

FIgURE 2: Titration curves for 10 mol \% LPA (O), DOPA (๑), and dehydroxy-LPA $(\triangle)$ in DOPC, relating bulk pH to chemical shift. Lines represent the nonlinear least-squares fits of the data to eq 1 (see Materials and Methods).

Table 1: Dissociation Constants for LPA, PA, and Dehydroxy-LPA in Extended Bilayers and Hexagonal $\mathrm{H}_{\mathrm{II}}$ Phase $^{a}$

\begin{tabular}{cccr}
\hline & LPA & PA & dehydroxy-LPA \\
\hline PC bilayer & & & \\
$\mathrm{p} K_{\mathrm{a} 1}$ & $2.9 \pm 0.3$ & $3.2 \pm 0.3$ & $3.4 \pm 0.15$ \\
$\mathrm{p} K_{\mathrm{a} 2}$ & $7.47 \pm 0.03$ & $7.92 \pm 0.03$ & $7.99 \pm 0.03$ \\
$\mathrm{PE} \mathrm{bilayer}$ & & & \\
$\mathrm{p} K_{\mathrm{a} 1}$ & $\mathrm{nd}^{b}$ & $\mathrm{nd}^{b}$ & \\
$\mathrm{p} K_{\mathrm{a} 2}$ & $6.88 \pm 0.04$ & $6.89 \pm 0.05$ & \\
$\mathrm{PE} \mathrm{hexagonal} \mathrm{phase}$ & & & \\
$\mathrm{p} K_{\mathrm{a} 1}$ & $3.1 \pm 0.6$ & $3.2 \pm 0.4$ & \\
$\mathrm{p} K_{\mathrm{a} 2}$ & $6.60 \pm 0.04$ & $7.02 \pm 0.04$ & \\
\hline
\end{tabular}

${ }^{a}$ The PE bilayer system contained $25 \mathrm{~mol} \% \mathrm{LPC}$ to force PE into a bilayer organization (for details, see the text). The LPA, PA, and dehydroxy-LPA content was $10 \mathrm{~mol} \%$ in all systems except for the PE hexagonal phase, where the LPA content was $5 \mathrm{~mol} \%$. ${ }^{b}$ Not determined.

between LPA and PA was maintained in the presence of physiological concentrations of $\mathrm{Mg}^{2+}$ (free $\mathrm{Mg}^{2+}$ concentration of $\sim 1 \mathrm{mM}$; data not shown). Although $\mathrm{Mg}^{2+}$ increased the charge of both LPA and PA, most likely by decreasing the proton concentration at the membrane interface, both lipids were affected to the same extent, and a substantial difference in charge between PA and LPA remained. The striking difference in ionization between PA and LPA must be related to the chemical structure of the backbone, all other conditions being the same [i.e., lipid background, (L)PA concentration, and ionic strength]. Therefore, we determined the titration behavior of a LPA compound lacking the free hydroxyl group at the $s n-2$ position of the glycerol backbone (dehydroxy-LPA). The dissociation behavior of dehydroxyLPA was essentially identical to that of PA (see Figure 2 and Table 1), demonstrating that the hydroxyl group of the LPA backbone is responsible for the difference in ionization behavior between LPA and PA. The difference in ionization behavior between LPA, on one hand, and dehydroxy-LPA and PA, on the other hand, could potentially be related to a difference in the orientation of the headgroup. To examine this possibility, we determined the residual chemical shift anisotropy of LPA and dehydroxy-LPA (10 mol \% in DOPC at $\mathrm{pH}$ 7.2) and found it to be very similar (data not shown). This result indicated that the conformation of dehydroxy- 

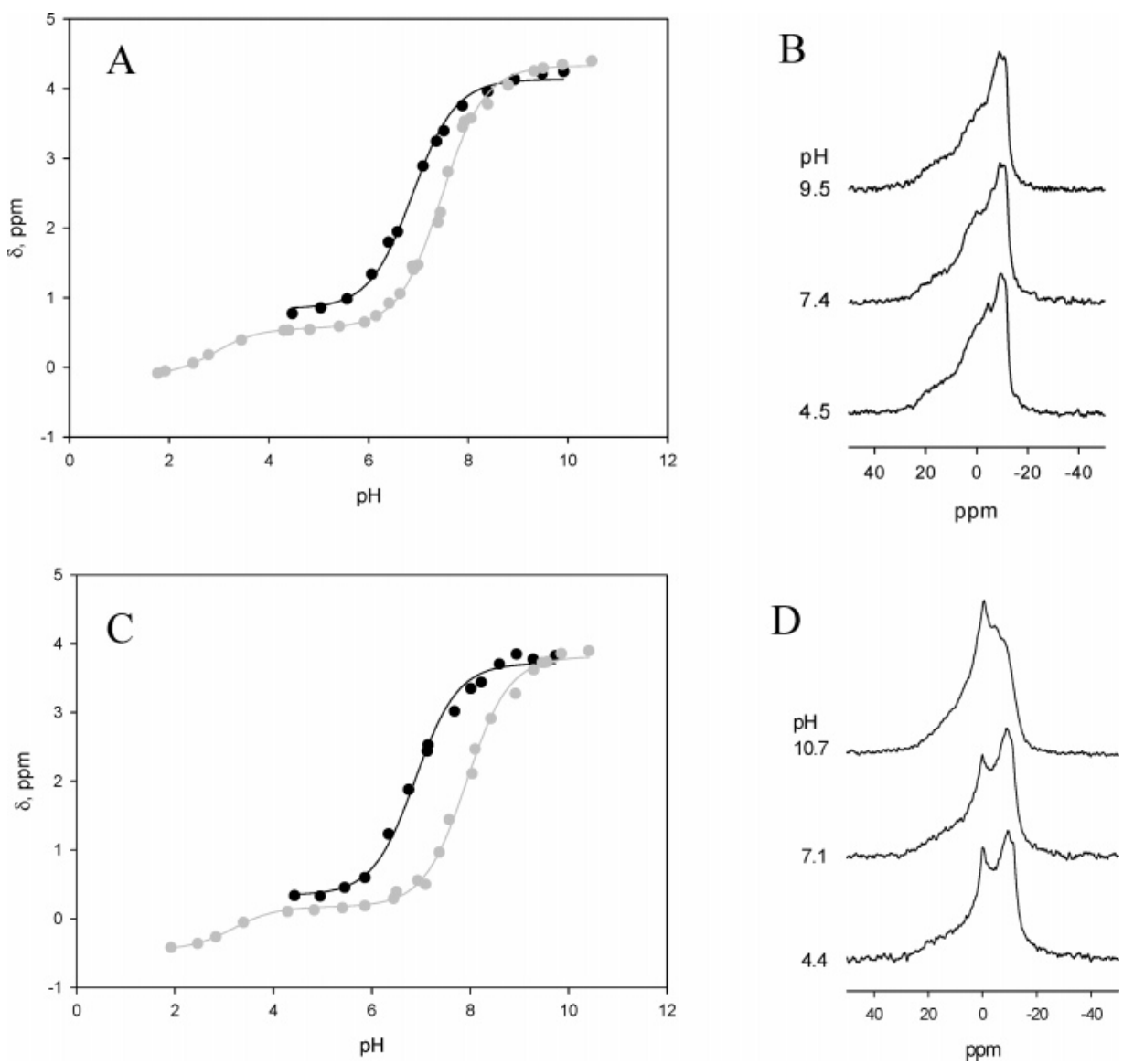

FIGURE 3: Titration curves for 10 mol \% LPA (A) and 10 mol \% DOPA (C) in PE/LPC (65:25) bilayers (black circles) and PC bilayers (gray circles; data from Figure 1). Lines represent the nonlinear least-squares fits to eq 1 (gray line) or 2 (black line). Representative static spectra at three representative $\mathrm{pH}$ values for $10 \mathrm{~mol} \%$ LPA (B) and $10 \mathrm{~mol} \%$ DOPA (D) in PE/LPC (65:25) bilayers.

LPA is close to that of LPA, and strongly suggested that the difference in ionization behavior was not related to a difference in the orientation of the headgroup but to a property of the hydroxyl group at the $s n-2$ position of LPA.

Dissociation Constants of $(L) P A$ in Bilayers and the Hexagonal $H_{I I}$ Phase of PE. Another major cellular membrane phospholipid is phosphatidylethanolamine (PE). $\mathrm{PE}$ is zwitterionic, like PC, but its headgroup is much smaller and carries a protonatable primary instead of a nonprotonatable quaternary amine. To test whether PE affects the protonation behavior of (L)PA, we first designed an experimental system rich in unsaturated $\mathrm{PE}$, but organized in a bilayer. Aqueous dispersions of dioleoyl-PE do not form bilayers at room temperature but prefer organization in an inverted $\mathrm{H}_{\text {II }}$ phase due to the cone shape of this PE [bilayer to inverted hexagonal, $\mathrm{L}_{\alpha}-\mathrm{H}_{\mathrm{II}}$, phase transition temperature of $\sim 3{ }^{\circ} \mathrm{C}$ $(25,26)]$. However, bilayers are formed if an appropriate amount of lysoPC (LPC) is added to complement the cone shape of PE (11, 27-29).

A low-field shoulder and a high-field peak characterize static ${ }^{31} \mathrm{P}$ NMR spectra of lipid mixtures organized in a fluid bilayer (30). Mixtures of $65 \mathrm{~mol} \% \mathrm{PE}, 25 \mathrm{~mol} \% \mathrm{LPC}$, and $10 \mathrm{~mol} \%$ LPA or PA were analyzed over a range of $\mathrm{pH}$ values $(4.0<\mathrm{pH}<10.5)$, and the ${ }^{31} \mathrm{P}$ NMR spectra confirmed an organization in fluid lipid bilayers over the complete $\mathrm{pH}$ range (Figure 3B,D). Occasionally a minor peak was present at the isotropic position (around $0 \mathrm{ppm}$ ), most likely originating from small vesicles. These PE/LPC bilayer systems were used for MAS NMR analysis, and will be termed "PE bilayer" throughout the rest of this paper. Results are shown in panels A and C of Figure 3 for LPA and PA, respectively (black lines); for comparison, the titration curves of (L)PA in a PC bilayer are also shown (gray lines). When the PE bilayer system is compared to the PC bilayer system, the titration curves for both LPA and PA are clearly shifted to lower $\mathrm{pH}$ values. This is reflected in the $\mathrm{p} K_{\mathrm{a} 2}$ for (L)PA that is significantly lower in the PE bilayer than in the PC bilayer (Table 1). Interestingly, the dissociation constants $\left(\mathrm{p} K_{\mathrm{a} 2}\right)$ of LPA and PA in this PE bilayer are now identical within experimental error (Table 1). These data clearly demonstrate that the charge on PA and LPA in extended bilayers is sensitive to the lipid composition. The observed effect of lipid composition on the ionization of (L)PA may be due either to a difference in lipid packing or, more likely, to a difference in the chemical properties of PC and PE.

To gain further insight into these possibilities, we determined the dissociation constants of (L)PA in a pure DOPE matrix which is organized in a hexagonal $\mathrm{H}_{\text {II }}$ phase at 20 ${ }^{\circ} \mathrm{C}$. Also, in the presence of $5 \mathrm{~mol} \%$ LPA [a type I lipid (12)], a hexagonal phase was formed as determined by broad line ${ }^{31} \mathrm{P}$ NMR (data not shown). Titration curves for $5 \mathrm{~mol}$ $\%$ LPA and $10 \mathrm{~mol} \% \mathrm{PA}$ in a PE hexagonal phase were recorded by MAS ${ }^{31} \mathrm{P}$ NMR. The chemical shift position of the PE peak was virtually constant except at high $\mathrm{pH}$, where it moved slightly to downfield values similar to what was observed in the PE bilayer system (data not shown). From the titration behavior of (L)PA, the dissociation constants were determined, and the results are given in Table 1. The 
$\mathrm{p} K_{\mathrm{a} 2}$ of PA is higher than that of LPA in the PE hexagonal phase, similar to the situation observed in the PC bilayer. Importantly, the $\mathrm{p} K_{\mathrm{a} 2}$ for (L)PA in the PE hexagonal phase is close to that found for (L)PA in the PE bilayer, but clearly deviates from that in PC bilayers, suggesting that indeed a difference in chemical properties between PE and PC is responsible for the different titration behavior of (L)PA in PE compared to PC. Since lipid packing is very different in a bilayer and $\mathrm{H}_{\mathrm{II}}$ phase (31), lipid packing apparently does not have a major effect on the ionization of (L)PA. The difference in the behavior of the PC and PE bilayer must thus be due to differences in their headgroup.

Headgroup Charge of (L)PA as a Function of PC:PE Ratio. To gain further insight into this headgroup specificity, we measured the ${ }^{31} \mathrm{P}$ chemical shift of $10 \mathrm{~mol} \%$ (L)PA in extended bilayers containing $\mathrm{PC}$ and $\mathrm{PE}$ at physiological $\mathrm{pH}$ ( $\mathrm{pH} 7.2$ ), while varying the PC:PE ratio.

Figure 4A shows representative MAS ${ }^{31} \mathrm{P}$ NMR spectra for LPA-containing samples, and the chemical shift positions of LPA, PE, and PC were clearly resolved (as indicated in Figure 4A). Indeed, an increase in PE concentration (decrease in PC concentration) resulted in a large downfield shift of the LPA peak corresponding to an increase in the charge for the LPA headgroup. These results are quantified in Figure 4B, which also includes a similar titration curve for PA. The ${ }^{31} \mathrm{P}$ chemical shift of (L)PA is plotted as a function of the PC:PE ratio, and the value of the chemical shift for LPA and PA in the pure PC bilayer is indicated by the dashed and dotted asymptotes, respectively (infinite PC:PE ratio). The large downfield change in the chemical shift of both LPA and PA shows that the ionization of (L)PA is indeed strongly enhanced with an increase in PE content (decrease in the PC:PE ratio).

It is well-known that intracellular membranes vary greatly in lipid composition with a large difference in the PC:PE ratio, in particular between the cytoplasmic and luminal leaflet of the Golgi membrane, and between the ER and cytoplasmic leaflet of the plasma membrane (32). To examine whether the charge on (L)PA varies between physiologically relevant phospholipid compositions, we measured the chemical shift of PA at physiological cytosolic $\mathrm{pH}(\mathrm{pH}$ 7.2) for phospholipid mixtures mimicking the ER and cytoplasmic leaflet of the PM. At these locations, (L)$\mathrm{PA}$ is involved in lipid synthesis and signaling. Lipid compositions are based on those of rat liver ER and plasma membrane (33), assuming a symmetric transbilayer lipid distribution for the ER, and an asymmetric transbilayer distribution in the plasma membrane identical to that of human erythrocytes (34). Minor lipids at these locations such as sphingomyelin and phosphoinositides were not included in these mixtures. The lipid mixtures mimicking the cytoplasmic leaflet of the PM and the ER membrane had PC: PE:PS:PA molar ratios of $1: 2: 2: 0.26$ and 3:1:0.7:0.25, respectively. Figure 5A shows representative MAS ${ }^{31} \mathrm{P}$ NMR spectra, with PC, PE/PS, and PA peaks indicated. The chemical shift position of PA in the PM lipid mixture is shifted downfield by $\sim 0.7 \mathrm{ppm}$ as compared to its position in the ER lipid mixture, supporting the hypothesis that the $\mathrm{PC}: \mathrm{PE}$ ratio is a major determinant of PA charge.

The relation between the chemical shift difference and the PE content of the two samples was somewhat masked by the differences in PS content. PS as an anionic lipid will
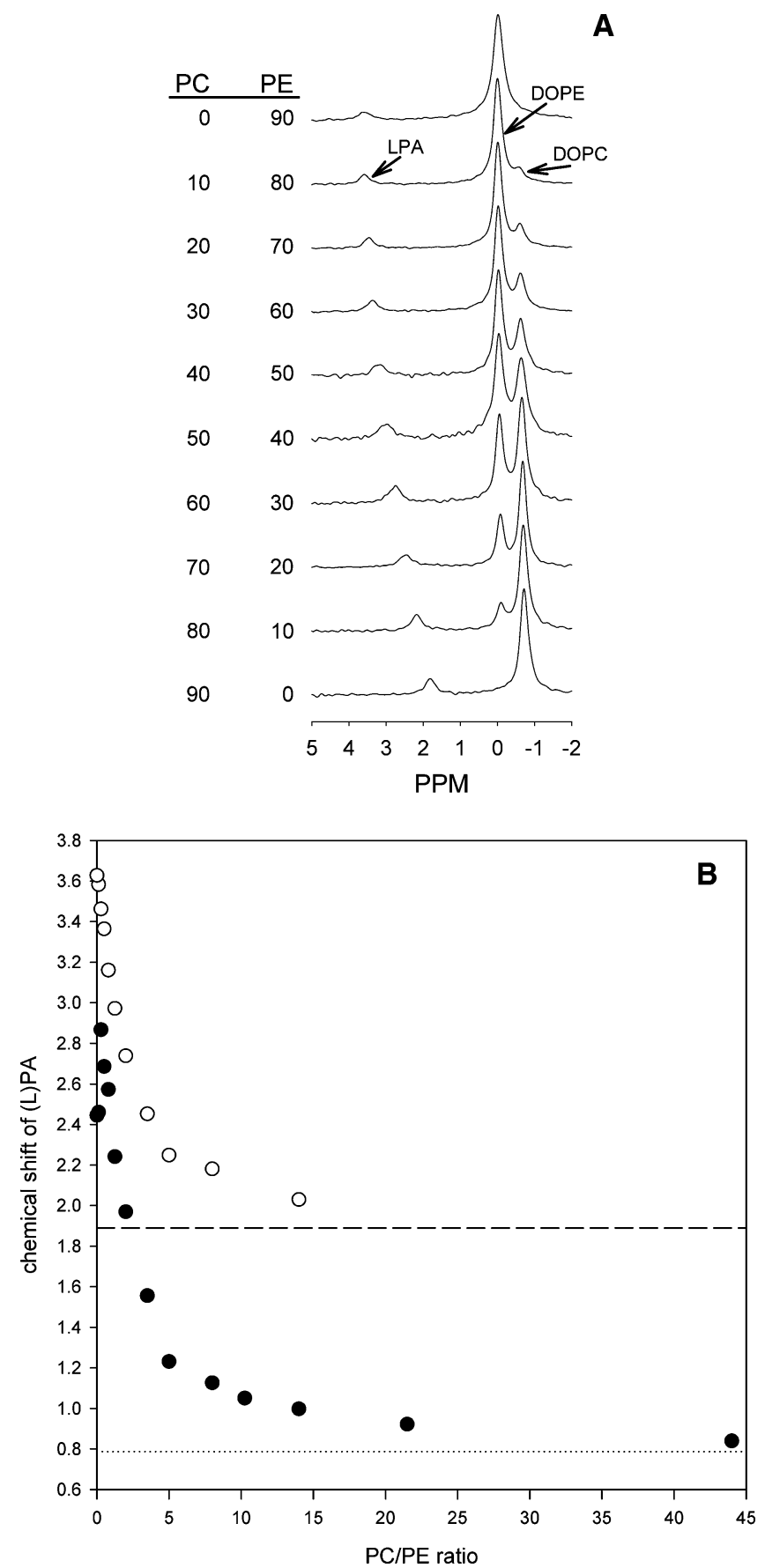

FIGURE 4: (A) Solid-state MAS ${ }^{31} \mathrm{P}$ NMR spectra for $10 \mathrm{~mol} \%$ LPA as a function of PC:PE ratio at $\mathrm{pH} 7.20 \pm 0.05$. In panel $\mathrm{B}$, the chemical shift of LPA $(O)$ and PA $(-)$ at $\mathrm{pH} 7.20 \pm 0.05$ is plotted as a function of the PC:PE molar ratio. The dotted and dashed lines represent the values of the chemical shift in pure PC, for $10 \mathrm{~mol} \%$ PA and LPA, respectively (infinite PC:PE ratio).

increase the proton concentration at the membrane interface, which in turn will affect the ionization of PA. Figure 5B indeed shows the expected PS dependence of the chemical shift of PA but also demonstrates that at a constant PS concentration, ionization of PA is strongly PE dependent.

In a similar experiment, the effects of cholesterol were examined (data not shown). At a high PE content (PC:PE molar ratio of 0.5$), 20 \mathrm{~mol} \%$ cholesterol had virtually no effect on the ionization of PA. At low PE concentrations (PC:PE molar ratio of 2), cholesterol induced a larger increase in the negative charge of PA, but the effect was small compared to that induced by $\mathrm{PE}(<50 \%$ of the 


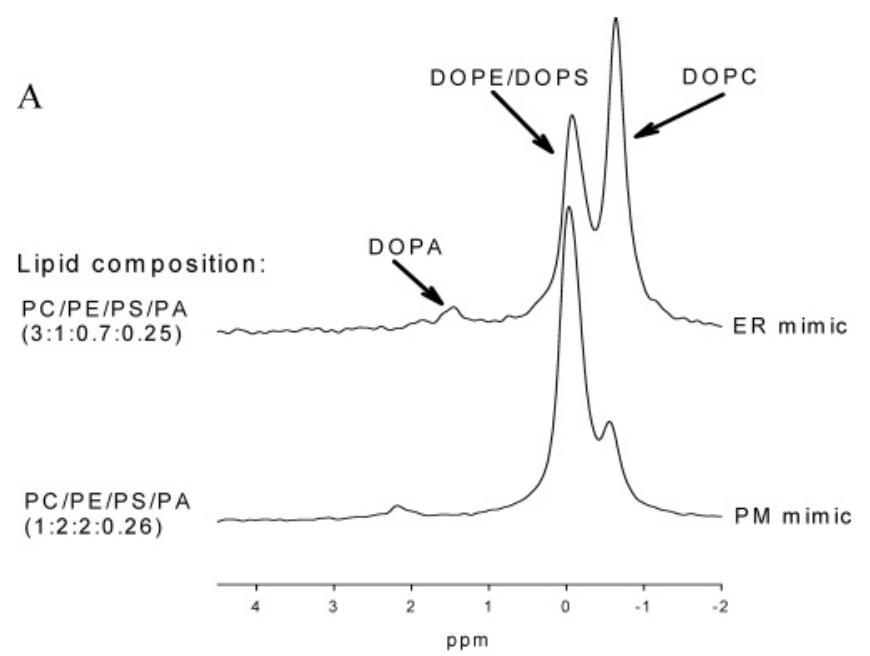

B

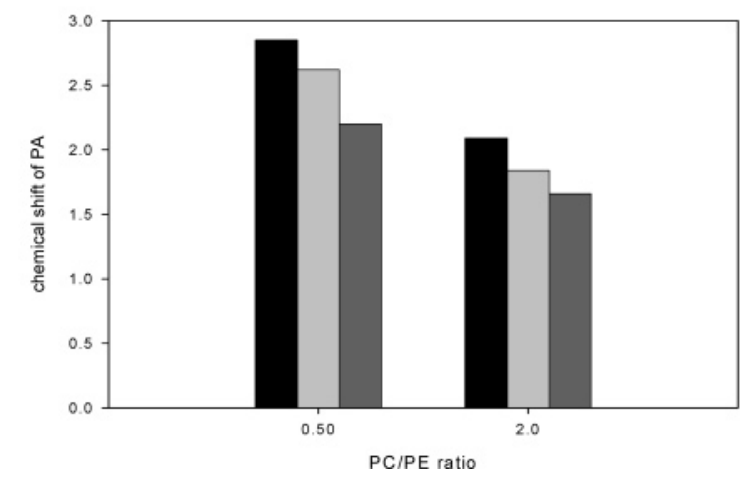

FIGURE 5: (A) Solid-state MAS ${ }^{31} \mathrm{P}$ NMR spectra for $5 \mathrm{~mol} \%$ PA in lipid mixtures mimicking the cytoplasmic leaflet of the plasma membrane (bottom curve) and ER membrane (top curve). (B) Chemical shift for $5 \mathrm{~mol} \% \mathrm{PA}$ in bilayers with a PC:PE molar ratio of 0.5 and 2.0 at a PS concentration of 0 (black), 10 (light gray), and $20 \mathrm{~mol} \%$ (dark gray).

chemical shift induced by adding $20 \mathrm{~mol} \% \mathrm{PE}$ to the same $\mathrm{PC} / \mathrm{PE}$ mixture). Thus, the $\mathrm{PC}: \mathrm{PE}$ ratio is a major determinant of PA charge, while cholesterol has only a weak effect on the ionization of PA.

\section{DISCUSSION}

In this study, we have determined by MAS ${ }^{31} \mathrm{P}$ NMR the dissociation constants of LPA and PA in bilayers of the zwitterionic phospholipids PC and PE. The second $\mathrm{p} K_{\mathrm{a}}$ of LPA, which falls in the physiological range, was found to be lower than that of PA, and we showed that it is the free hydroxyl on the LPA backbone that is responsible for this difference in ionization behavior. Furthermore, the degree of ionization of both LPA and PA was found to depend strongly on bilayer lipid composition, i.e., the PC:PE ratio, of the membrane. These data will be discussed in terms of the unique hydrogen bonding possibilities of the phosphomonoester headgroup of (L)PA.

First, we will briefly discuss the factors influencing phospholipid ionization (for more comprehensive reviews, see refs 16,17 , and 35). Phospholipid protonation in a bilayer membrane deviates from that of the free molecule in solution. The main reason for this difference is the membrane-water interface. Protonation depends on the local $\mathrm{pH}$ at this interface, and this (intrinsic) $\mathrm{pH}$ can be dramatically different from that found in solution. Introducing an overall negative charge in the membrane, for instance, by incorporating PS, increases the proton concentration at the lipid-water interface by attracting protons from the bulk solution, thereby lowering the intrinsic $\mathrm{pH}$ and increasing the apparent $\mathrm{p} K_{\mathrm{a}}$ of a protonatable group. Similarly, the presence of a positive charge, as, for instance, found in the zwitterionic lipid PC, increases the local $\mathrm{pH}$ at the lipid headgroup and decreases the apparent $\mathrm{p} K_{\mathrm{a}}$ of the phosphate of the same molecule. Indeed, PS decreases the charge of PA as shown in Figure $5 \mathrm{~B}$, and the $\mathrm{p} K_{\mathrm{a}}$ of the phosphate of $\mathrm{PC}$ is well below that of the first $\mathrm{p} K_{\mathrm{a}}$ of (L)PA as shown in Figure 1.

The $\mathrm{p} K_{\mathrm{a}}$ of a protonatable group can also be affected by the formation of hydrogen bonds. A proton that is participating in a hydrogen bond will dissociate less easily, resulting in a higher $\mathrm{p} K_{\mathrm{a}}$ for the protonatable group (36-38).

Ionization Behavior of LPA and PA in Extended Phospholipid Bilayers. The dissociation constants of $10 \mathrm{~mol} \%$ PA and LPA were measured in a PC bilayer. Interestingly, we find that the second $\mathrm{p} K_{\mathrm{a}}$ differs significantly between the two lipids, despite identical headgroups. The $\mathrm{p} K_{\mathrm{a} 2}$ of LPA is $0.45 \pm 0.06 \mathrm{pH}$ unit lower than that of PA.

This difference in dissociation constant must be due to an intrinsic difference between PA and LPA, and we provided direct evidence that the hydroxyl group at the $s n-2$ position of the backbone of LPA is responsible for this difference (see Figure 2). How is this hydroxyl group able to lower the second $\mathrm{p} K_{\mathrm{a}}$ of LPA with respect to PA? This is most likely not due to a difference in headgroup orientation. The residual CSAs, a measure of lipid order and dynamics, of LPA and dehydroxy-LPA are similar, suggesting that the conformation of the headgroup in dehydroxy-LPA is close to that in LPA. In support of this, the orientation of the glycerol backbone in LPA and a dehydroxy-LPA-related compound (acyl chain attached via an ether instead of an ester linkage) was found to be nearly identical [but very different from that of PA (39-41)]. Instead, we propose that the difference in ionization of LPA and PA is the result of an intramolecular hydrogen bond between the 2-hydroxyl and the deprotonated headgroup in LPA. Such a hydrogen bond is indeed observed in the LPA crystal structure (40). Apparently, this intramolecular hydrogen bond is a special feature of LPA that is preserved in excess water. Intramolecular hydrogen bonds are known to influence the (de)protonation behavior of phospholipids. Haines and co-workers showed for cardiolipin (CL; see Figure 6A) that the $\mathrm{p} K_{\mathrm{a}}$ 's for the two hydroxyls in the headgroup of CL are not identical $(37,42)$. The authors also showed that this is due to the hydroxyl on the glycerol connecting the two phosphates of CL, and proposed that as soon as one of the two hydroxyls has dissociated its proton it is able to form a hydrogen bond network (see Figure 6A), which stabilizes the proton on the other hydroxyl. In analogy to $\mathrm{CL}$, we propose that at near-physiological $\mathrm{pH}$ both PA and LPA are able to form an intramolecular hydrogen bond between the deprotonated and protonated hydroxyl of their phosphomonoester headgroup (Figure 6B), a feature unique to the phosphomonoester. This intramolecular hydrogen bond stabilizes the second proton of the phosphomonoester and would therefore increase $\mathrm{p} K_{\mathrm{a} 2}$. However, LPA also has the possibility to form an intramolecular hydrogen bond between the hydroxyl at the $s n-2$ position of its glycerol backbone and the deprotonated hydroxyl of the phosphomonoester headgroup (Figure 6B), destabilizing the second proton and resulting in a lower $\mathrm{p} K_{\mathrm{a} 2}$ (and a charge more negative than 
A<smiles>O=C1OCC23COP(=O)(O[Na])O[C@@H]2OP(=O)(OC3)OO1</smiles>

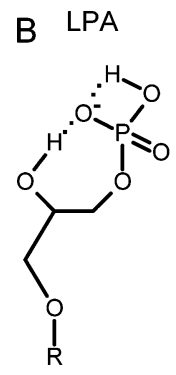
dehydroxy-LPA

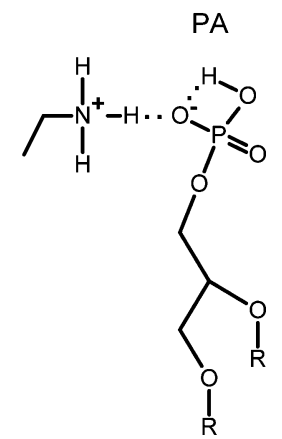

FIGURE 6: (A) Chemical structure of the headgroup of cardiolipin (DAG, diacylglycerol) when one (left) of the two phosphodiesters has dissociated its proton; hydrogen bonds are represented by two dots (adapted from refs 37 and 42). (B) Chemical structure of LPA (left), dehydroxy-LPA (middle), and PA (right) when carrying one negative charge. R stands for an oleoyl (18:1) acyl chain, and the primary amine group to the left of PA represents part of a PE headgroup.

that of PA at the same $\mathrm{pH})$. Indeed, the LPA compound lacking the hydroxyl at the $s n-2$ position, and thus unable to form this intramolecular hydrogen bond, behaves in a manner identical to that of PA. An important implication of this interpretation is that the phosphate of LPA is localized within hydrogen bonding distance of the hydroxyl at the $s n-2$ position of the glycerol backbone.

This hydrogen bond model also provides an explanation for the other major observation in our study, namely, that PE facilitates deprotonation of (L)PA in a bilayer. As opposed to the quaternary amine of PC, PE carries a primary amine group that is able to efficiently donate protons to form hydrogen bonds (see Figure 6B). Moreover, the headgroup of PE lies more or less parallel to the membrane surface (43), and the positively charged amine is therefore uniquely positioned to interact electrostatically with the phosphate (when it is negatively charged) of the (L)PA headgroup bringing both sufficiently close in space to allow hydrogen bond formation. This intermolecular hydrogen bond will destabilize the intramolecular hydrogen bond within the phosphomonoester headgroup, and thus lower the second dissociation constant.

Our model also explains the observation that LPA and PA have essentially the same $\mathrm{p} K_{\mathrm{a} 2}$ in a bilayer rich in PE. The large amount of hydrogen bond donors provided by the amine group of PE will overrule the hydrogen bonding ability of the hydroxyl at the $s n-2$ position of LPA.

We also noticed a small contribution of lipid packing on the ionization of PA upon a change from the PE bilayer to the PE hexagonal phase, as indicated by a slight increase in $\mathrm{p} K_{\mathrm{a} 2}$ (see Table 1). We interpret this result in terms of curvature. Lipid headgroups in the hexagonal phase are more tightly packed than in a flat lipid bilayer due to the concave (negative) curvature of this lipid organization. This tighter headgroup packing is only possible at a lower PA charge, reducing the effective headgroup area. These results agree well with previous observations describing the effects of lipid packing on PA charge $(20,21)$, and also explain the effects of cholesterol on the ionization of PA. Cholesterol has a negative spontaneous curvature close to that of DOPE (44), and the small increase in the negative charge of PA that we observed in the presence of cholesterol can be explained by its effects on lipid packing. An alternative explanation, the formation of a hydrogen bond between the $\mathrm{OH}$ group of cholesterol and PA, is unlikely given the position of the $\mathrm{OH}$ group within the lipid bilayer. Instead, cholesterol appears to form a hydrogen bond with the carbonyl oxygen of the ester-bound acyl chains (45); similar hydrogen bonds have been suggested for the $\mathrm{OH}$ group of sphingomyelin (36).

Biological Implications. The phosphomonoester headgroup of (L)PA with its unique intra- and intermolecular hydrogen bond capabilities is what makes these simple but key phospholipids so special. For example, our observation that LPA carries more charge than PA in a PC bilayer at the same (physiological) $\mathrm{pH}$ implies that the metabolic interconversion of these lipids by, for example, $\mathrm{PLA}_{1}$ and $\mathrm{PLA}_{2}$ $(\mathrm{PA} \rightarrow$ LPA) or LPA acyltransferases (LPAATs) such as $\mathrm{CtBP}_{3} / \mathrm{BARS}$ and endophilin (LPA $\rightarrow$ PA) will affect local membrane charge if the membrane has a sufficiently high PC:PE ratio. This charge difference between LPA and PA is maintained in the presence of physiological (cytosolic) concentrations of $\mathrm{Mg}^{2+}$, and may well have important consequences for lipid and lipid-protein interactions. The intramolecular hydrogen bond between the headgroup of LPA and the hydroxyl on the glycerol backbone is also likely to contribute to specific recognition of LPA by LPA-binding proteins such as the LPA receptors.

In addition, our observation that the deprotonation of (L)PA depends on the local PC:PE ratio and should thus be different in the different organelles of a eukaryotic cell may have important implications for (L)PA-protein interactions at these subcellular locations. The specific recruitment of downstream effectors to PA at a particular intracellular site might, in part, rely on the deprotonation behavior of PA at this site. In yeast, for example, the protein Opi1 is retained by PA at the ER and not elsewhere in the cell (46), whereas Raf-1 kinase is specifically recruited by PA to the plasma membrane $(47,48)$. Our data also imply that hydrogen bonds between PA and basic residues such as lysines and arginines in proteins probably play an important role in protein-lipid interactions. Indeed, we have strong indications for such hydrogen bonds in model membrane experiments (manuscript in preparation).

Finally, modulation of headgroup charge may be part of the mechanism by which (L)PA regulates biomembrane fusion and fission events. For example, PA formed in a budding transport carrier at the Golgi by $\mathrm{CtBP}_{3} / \mathrm{BARS}$ or diacylglycerol kinase will carry more charge in the cytoplasmic leaflet than in the luminal leaflet of the Golgi due to the large difference in the PC:PE ratio ( $\sim 8$-fold lower in the cytoplasmic leaflet) and $\mathrm{pH}(\sim 7.2$ at the cytoplasmic leaflet and $\sim 6.0$ in the Golgi lumen). This would result in PA with more negative curvature in the Golgi luminal leaflet than in the cytoplasmic leaflet. If PA formed in the cytoplasmic leaflet would be able to translocate (49) to the luminal site, it might facilitate the fission of the budding transport carrier by CtBP3/BARS $(11,50,51)$. Thus, a defined charge of (L)PA at a particular (intra)cellular location, determined largely by the local PC:PE ratio, may be a key element of the action of (L)PA at that specific 
location, and regulate protein recruitment, activation, etc. Our observations on the unique ionization properties of LPA and PA may well be the basis of their special functions in the cell.

\section{ACKNOWLEDGMENT}

We thank Antoinette Killian, Eefjan Breukink, and Mandy Lutters for helpful discussions and practical assistance.

\section{REFERENCES}

1. Athenstaedt, K., and Daum, G. (1999) Phosphatidic acid, a key intermediate in lipid metabolism, Eur. J. Biochem. 266, 1-16.

2. Contos, J. J., Ishii, I., and Chun, J. (2000) Lysophosphatidic acid receptors, Mol. Pharmacol. 58, 1188-96.

3. Tigyi, G., and Parrill, A. L. (2003) Molecular mechanisms of lysophosphatidic acid action, Prog. Lipid Res. 42, 498-526.

4. Ishii, I., Fukushima, N., Ye, X., and Chun, J. (2004) Lysophospholipid receptors: Signaling and biology, Annu. Rev. Biochem. $73,321-54$.

5. Siddhanta, A., and Shields, D. (1998) Secretory vesicle budding from the trans-Golgi network is mediated by phosphatidic acid levels, J. Biol. Chem. 273, 17995-8.

6. Roth, M. G., Bi, K., Ktistakis, N. T., and Yu, S. (1999) Phospholipase D as an effector for ADP-ribosylation factor in the regulation of vesicular traffic, Chem. Phys. Lipids 98, 141-52.

7. Schmidt, A., Wolde, M., Thiele, C., Fest, W., Kratzin, H., Podtelejnikov, A. V., Witke, W., Huttner, W. B., and Söling, H. D. (1999) Endophilin I mediates synaptic vesicle formation by transfer of arachidonate to lysophosphatidic acid, Nature 401, $133-41$

8. Weigert, R., Silletta, M. G., Spano, S., Turacchio, G., Cericola, C., Colanzi, A., Senatore, S., Mancini, R., Polishchuk, E. V., Salmona, M., Facchiano, F., Burger, K. N. J., Mironov, A., Luini, A., and Corda, D. (1999) CtBP/BARS induces fission of Golgi membranes by acylating lysophosphatidic acid, Nature 402, 42933.

9. Bankaitis, V. A. (2002) Cell biology. Slick recruitment to the Golgi, Science 295, 290-1.

10. Karbowski, M., Jeong, S. Y., and Youle, R. J. (2004) Endophilin $\mathrm{B} 1$ is required for the maintenance of mitochondrial morphology, J. Cell Biol. 166, 1027-39.

11. Kooijman, E. E., Chupin, V., de Kruijff, B., and Burger, K. N. J. (2003) Modulation of membrane curvature by phosphatidic acid and lysophosphatidic acid, Traffic 4, 162-74.

12. Kooijman, E. E., Chupin, V., Fuller, N. L., Kozlov, M. M., de Kruijff, B., Burger, K. N. J., and Rand, P. R. (2005) Spontaneous curvature of phosphatidic acid and lysophosphatidic acid, Biochemistry 44, 2097-102.

13. Testerink, C., and Munnik, T. (2005) Phosphatidic acid: A multifunctional stress signaling lipid in plants, Trends Plant Sci. $10,368-75$.

14. Verkleij, A. J., De Maagd, R., Leunissen-Bijvelt, J., and De Kruijff, B. (1982) Divalent cations and chlorpromazine can induce nonbilayer structures in phosphatidic acid-containing model membranes, Biochim. Biophys. Acta 684, 255-62.

15. Farren, S. B., Hope, M. J., and Cullis, P. R. (1983) Polymorphic phase preferences of phosphatidic acid: $\mathrm{A}^{31} \mathrm{P}$ and ${ }^{2} \mathrm{H}$ NMR study, Biochem. Biophys. Res. Commun. 111, 675-82.

16. Cevc, G. (1990) Membrane electrostatics, Biochim. Biophys. Acta $1031,311-82$.

17. Tocanne, J. F., and Teissie, J. (1990) Ionization of phospholipids and phospholipid-supported interfacial lateral diffusion of protons in membrane model systems, Biochim. Biophys. Acta 1031, 11142.

18. Koter, M., de Kruijff, B., and van Deenen, L. L. (1978) Calciuminduced aggregation and fusion of mixed phosphatidylcholinephosphatidic acid vesicles as studied by ${ }^{31} \mathrm{P}$ NMR, Biochim. Biophys. Acta 514, 255-63.

19. Hauser, H. (1989) Mechanism of spontaneous vesiculation, Proc. Natl. Acad. Sci. U.S.A. 86, 5351-5.

20. Swairjo, M. A., Seaton, B. A., and Roberts, M. F. (1994) Effect of vesicle composition and curvature on the dissociation of phosphatidic acid in small unilamellar vesicles: $\mathrm{A}^{31} \mathrm{P}$ NMR study, Biochim. Biophys. Acta 1191, 354-61.
21. Traikia, M., Warschawski, D. E., Lambert, O., Rigaud, J. L., and Devaux, P. F. (2002) Asymmetrical membranes and surface tension, Biophys. J. 83, 1443-54.

22. Lynch, K. R., Hopper, D. W., Carlisle, S. J., Catalano, J. G., Zhang, M., and MacDonald, T. L. (1997) Structure/activity relationships in lysophosphatidic acid: The 2-hydroxyl moiety, Mol. Pharmacol. 52, 75-81.

23. Rouser, G., Fleischer, S., and Yamamoto, A. (1970) Twodimensional thin layer chromatographic separation of polar lipids and determination of phospholipids by phosphorus analysis of spots, Lipids 5, 494-6.

24. Appleton, T. G., Hall, J. R., Ralph, S. F., and Thompson, C. S. M. (1989) NMR Study of Acid-Base Equilibria and Other Reactions of Ammineplatinum Complexes with Aqua and Hydroxo Ligands, Inorg. Chem. 28, 1989-93.

25. Tate, M. W., and Gruner, S. M. (1987) Lipid polymorphism of mixtures of dioleoylphosphatidylethanolamine and saturated and monounsaturated phosphatidylcholines of various chain lengths, Biochemistry 26, 231-6.

26. Toombes, G. E., Finnefrock, A. C., Tate, M. W., and Gruner, S. M. (2002) Determination of $\mathrm{L}(\alpha)-\mathrm{H}(\mathrm{II})$ phase transition temperature for 1,2-dioleoyl-sn-glycero-3-phosphatidylethanolamine, Biophys. J. 82, 2504-10.

27. Gruner, S. M., Cullis, P. R., Hope, M. J., and Tilcock, C. P. (1985) Lipid polymorphism: The molecular basis of nonbilayer phases, Annu. Rev. Biophys. Biophys. Chem. 14, 211-38.

28. Janes, N. (1996) Curvature stress and polymorphism in membranes, Chem. Phys. Lipids 81, 133-50.

29. Fuller, N., and Rand, R. P. (2001) The influence of lysolipids on the spontaneous curvature and bending elasticity of phospholipid membranes, Biophys. J. 81, 243-54.

30. Cullis, P. R., and de Kruijff, B. (1979) Lipid polymorphism and the functional roles of lipids in biological membranes, Biochim. Biophys. Acta 559, 399-420.

31. Lafleur, M., Bloom, M., and Cullis, P. R. (1990) Lipid polymorphism and hydrocarbon order, Biochem. Cell Biol. 68, 1-8.

32. van Meer, G., and Sprong, H. (2004) Membrane lipids and vesicular traffic, Curr. Opin. Cell Biol. 16, 373-8.

33. Fleischer, B., Zambrano, F., and Fleischer, S. (1974) Biochemical characterization of the golgi complex of mammalian cells, $J$. Supramol. Struct. 2, 737-50.

34. Verkleij, A. J., Zwaal, R. F., Roelofsen, B., Comfurius, P., Kastelijn, D., and van Deenen, L. L. (1973) The asymmetric distribution of phospholipids in the human red cell membrane. A combined study using phospholipases and freeze-etch electron microscopy, Biochim. Biophys. Acta 323, 178-93.

35. McLaughlin, S. (1989) The electrostatic properties of membranes, Annu. Rev. Biophys. Biophys. Chem. 18, 113-36.

36. Boggs, J. M. (1987) Lipid intermolecular hydrogen bonding: Influence on structural organization and membrane function, Biochim. Biophys. Acta 906, 353-404.

37. Kates, M., Syz, J. Y., Gosser, D., and Haines, T. H. (1993) pHdissociation characteristics of cardiolipin and its 2 -deoxy analogue, Lipids 28, 877-82.

38. Moncelli, M. R., Becucci, L., and Guidelli, R. (1994) The intrinsic $\mathrm{p} K_{\mathrm{a}}$ values for phosphatidylcholine, phosphatidylethanolamine, and phosphatidylserine in monolayers deposited on mercury electrodes, Biophys. J. 66, 1969-80.

39. Harlos, K., Eibl, H., Pascher, I., and Sundell, S. (1984) Conformation and packing properties of phosphatidic acid: The crystal structure of monosodium dimyristoylphosphatidate, Chem. Phys. Lipids 34, 115-26.

40. Pascher, I., and Sundell, S. (1985) Interactions and space requirements of the phosphate head group in membrane lipids. The crystal structure of disodium lysophosphatidate dihydrate, Chem. Phys. Lipids 37, 241-50.

41. Pascher, I., Sundell, S., Eibl, H., and Harlos, K. (1984) Interactions and space requirement of the phosphate head group of membrane lipids: The single-crystal structures of a triclinic and a monoclinic form of hexadecyl-2-deoxy-glycerophosphoric acid monohydrate, Chem. Phys. Lipids 35, 103-15.

42. Haines, T. H., and Dencher, N. A. (2002) Cardiolipin: A proton trap for oxidative phosphorylation, FEBS Lett. 528, 35-9.

43. Langner, M., and Kubica, K. (1999) The electrostatics of lipid surfaces, Chem. Phys. Lipids 101, 3-35. 
44. Chen, Z., and Rand, R. P. (1997) The influence of cholesterol on phospholipid membrane curvature and bending elasticity, Biophys. J. 73, 267-76.

45. Ramsammy, L. S., Volwerk, H., Lipton, L. C., and Brockerhoff, H. (1983) Association of cholesterol with lysophosphatidylcholine, Chem. Phys. Lipids 32, 83-9.

46. Loewen, C. J., Gaspar, M. L., Jesch, S. A., Delon, C., Ktistakis, N. T., Henry, S. A., and Levine, T. P. (2004) Phospholipid metabolism regulated by a transcription factor sensing phosphatidic acid, Science 304, 1644-7.

47. Ghosh, S., Strum, J. C., Sciorra, V. A., Daniel, L., and Bell, R. M. (1996) Raf-1 kinase possesses distinct binding domains for phosphatidylserine and phosphatidic acid. Phosphatidic acid regulates the translocation of Raf-1 in 12-O-tetradecanoylphorbol13-acetate-stimulated Madin-Darby canine kidney cells, J. Biol. Chem. 271, 8472-80.

48. Rizzo, M. A., Shome, K., Watkins, S. C., and Romero, G. (2000) The recruitment of Raf-1 to membranes is mediated by direct interaction with phosphatidic acid and is independent of association with Ras, J. Biol. Chem. 275, 23911-8.

49. Kol, M. A., van Laak, A. N., Rijkers, D. T., Killian, J. A., de Kroon, A. I., and de Kruijff, B. (2003) Phospholipid flop induced by transmembrane peptides in model membranes is modulated by lipid composition, Biochemistry 42, 231-7.

50. Bonazzi, M., Spano, S., Turacchio, G., Cericola, C., Valente, C., Colanzi, A., Kweon, H. S., Hsu, V. W., Polishchuck, E. V., Polishchuck, R. S., Sallese, M., Pulvirenti, T., Corda, D., and Luini, A. (2005) CtBP3/BARS drives membrane fission in dynamin-independent transport pathways, Nat. Cell Biol. 7, 57080.

51. Kozlovsky, Y., and Kozlov, M. M. (2003) Membrane fission: Model for intermediate structures, Biophys. J. 85, 85-96.

BI0518794 